\title{
Analisi termica per la valutazione del comportamento a fatica di provini soggetti a successive serie di carichi
}

\author{
Antonino Risitano \\ Università di Catania, Dipartimento d'Ingegneria Industriale e Meccanica; Viale A. Doria 6, 95125 Catania, Italia \\ arisitan@diim.unict.it \\ Giacomo Risitano \\ Università degli Studi Guglielmo Marconi, Facoltà di Scienze e Tecnologie Applicate; Via Plinio 44, 00193 Roma, Italia; \\ g.risitano@unimarconi.it
}

Riassunto. Partendo dalla osservazione che provini precedentemente danneggiati hanno un limite di fatica differente (vedi Miner) viene esaminato il comportamento di un acciaio soggetto a serie di carichi affaticanti al fine di verificare la conseguente risposta termica.

L'esame, mediante analisi termica, delle prove di fatica su provini di acciaio C40 danneggiati a diverso grado, da interessanti indicazioni sul ruolo dei carichi applicati in relazione alla possibilità di produrre danno nel materiale. Viene messo in evidenza come la temperatura, conseguenza dell'energia consumata dal provino, possa essere elemento indicativo dello stato del materiale. Vengono riportate le curve di fatica e le curve di temperatura di provini lisci sottoposti a storie di carico diverse. Da esse si può evincere che mentre è valida la legge di linearità del danno $\left(\sum_{\mathrm{i}} \mathrm{n}_{\mathrm{i}} / \mathrm{N}_{\mathrm{i}}=\right.$ cost $)$, la curva definita secondo la regola del Miner-Manson $\left(\sum_{\mathrm{i}} \mathrm{n}_{\mathrm{i}} / \mathrm{N}_{\mathrm{i}}=1\right)$, che tiene solo conto del numero di volte in cui la tensione supera il limite di fatica del materiale (provini lisci), invece, non sempre rispecchia il reale stato di danno. Questo specialmente quando si è in presenza di carichi prossimi al limite di fatica che, per la sequenza dell'applicazione, possono diventare carichi affaticanti.

ABSTRACT. Starting from the observation that previously damaged specimens have a fatigue limit differences (see Miner), the behavior of a steel, subject to series of fatigue loads, was examined to verify the resulting thermal response.

The review, by thermal analysis, fatigue tests on samples of steel C40 damaged in varying degrees, provides valuable insights on the role of loads applied in relation to the possibility of producing damage in the material. The temperature, therefore the energy consumed, is an element indicative of the state of the material. The fatigue curves and the curves of temperature of smooth specimens, subjected to different load histories have been reported. These are demonstrated that a valid law of linear damage $\left(\sum_{\mathrm{i}} \mathrm{n}_{\mathrm{i}} / \mathrm{N}_{\mathrm{i}}=\right.$ cost $)$, but the curve defined under the rule of Miner-Manson $\left(\sum_{\mathrm{i}} \mathrm{n}_{\mathrm{i}} / \mathrm{N}_{\mathrm{i}}=1\right)$, which takes into account only the number of times the stress exceeds the fatigue limit of the material (smooth specimens), however, does not always reflect the actual state of damage. Especially when the loads are close to the fatigue limit, for the sequence of the application, loads can become fatiguing.

Parole Chiave. Termografia; Limite di fatica; Teoria del danno; Miner. 


\section{INTRODUZIONE}

$\grave{\mathrm{E}}$ noto che la rottura per fatica avviene quando nel materiale la sollecitazione a livello locale supera certi valori per cui si generano deformazioni irreversibili. Durante questa fase come già osservato da Taylor e Quinney [1] la quasi totalità dell'energia è convertita in calore. In ogni caso, tale quantità di calore, e di conseguenza la temperatura, risulta funzione di un coefficiente $\beta$ dipendente, nel caso più generale, da diversi fattori: la deformazione plastica, l'entropia, la densità di dislocazione. Secondo Mason et Al. [2], per valori di tensione inferiori al limite di fatica (low stress region) l'energia del ciclo di isteresi è dovuta a meccanismi dissipativi "anelastici" che non procurano danni sul materiale. Per valori superiori al limite di fatica (high stress region) l'energia di isteresi è quella dovuta a deformazioni plastiche. Esiste una zona di transizione in cui l'energia del ciclo di isteresi si compone della parte anelastica e della parte plastica. Questa zona di transizione si può pensare corrispondente alla banda di dispersione relativa al limite di fatica. Constatato che per valori di tensione superiori al limite di fatica, l'energia in gioco dissipativa è quella corrispondente a deformazioni plastiche Feltner e Morrow [3] propongono un modello teorico per la valutazione del fenomeno e definiscono la legge che lega tensioni e numero di cicli. E' stato messo in evidenza anche che la rottura di un provino avviene quando l'energia totale di deformazione plastica per unità di volume raggiunge un valore $\mathrm{E}_{\mathrm{c}}$ limite, costante caratteristico di ciascun materiale. Nel caso di metalli, per valori sufficientemente elevati di deformazione locale rispetto a quella plastica a rottura (superiore a 0,15), il valore di tale coefficiente si può ritenere costante. Ne consegue che, quando si sollecitano acciai con tensioni al di sopra del limite di fatica, la parte di calore sviluppato per ciclo, è proporzionale all'energia di deformazione plastica per ciclo $\mathrm{E}_{\mathrm{p} \text {, }}$ di conseguenza, per provini dello stesso materiale e della stessa forma, e per valori non eccessivamente elevati di temperatura raggiunti dal provino (minore di $200^{\circ} \mathrm{C}$, in modo da poter trascurare l'effetto irraggiante), anche la temperatura si può porre proporzionale al lavoro di deformazione plastica $\mathrm{E}_{\mathrm{pi}}$ (plastic work). Dal punto di vista sperimentale è possibile notare che in prove di fatica, solo quando si è al di sopra del limite di fatica (quindi, in presenza di deformazioni plastiche locali) si notano sulla superficie del provino incrementi significativi della temperatura (Dengel e Harig [4] e Kaleta et Al. [5]). Ciò è giustificato, come già detto, dal fatto che per sollecitazioni al di sotto del limite di fatica, l'effetto dovuto allo smorzamento interno della fase elastica del materiale e a tutti i fenomeni reversibili, è praticamente trascurabile in confronto a quello che si manifesta allorquando compaiono le prime deformazioni irreversibili. Questa ultima osservazione ha permesso di sviluppare differenti metodologie per la ricerca del limite di fatica (Risitano, Luong, Curti, Blarasin, Amiri, etc.). E' stato visto sperimentalmente e dimostrato attraverso modelli teorici che in prove di fatica su acciai con sollecitazione al di sopra del limite di fatica, la temperatura superficiale del provino dopo un prima incremento iniziale (fatica a basso numero di cicli) si mantiene pressoché costante (fatica ad alto numero di cicli) fino a pochi cicli (circa 500) prima della completa rottura (fatica ad altissimo numero di cicli) del provino (Fig. 1).

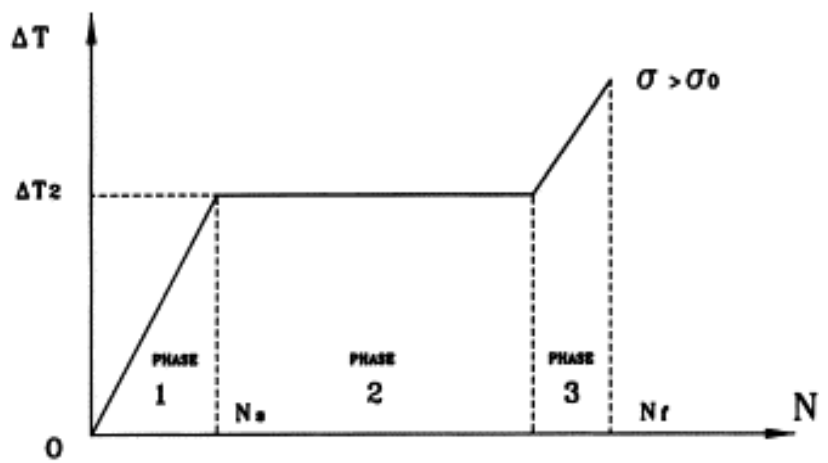

Figura 1: Incremento qualitativo della temperature in funzione del numero di cicli per una tensione sopra il limite di fatica $\Delta \sigma_{0}$.

Applicando quanto prima detto al volume elementare relativo alla zona di rottura, per cui con sistemi a largo campo è possibile prima seguire e poi analizzate l'evoluzione della temperatura, si vede che la quantità totale di calore sviluppato durante tutta la prova, a meno di una costante dipendente dal coefficiente globale di trasmissione del calore (conduzione, convenzione, irraggiamento) del materiale e della geometria del provino, risulta praticamente proporzionale all'integrale della temperatura durante tutto il tempo di prova (fino alla rottura) $\Phi=\int_{\mathrm{Ni}} \operatorname{Tdn}_{\mathrm{i}}$ (Fig. 2). Dal punto di vista pratico, per la costanza della temperatura durante quasi tutta la prova (Arig, Risitano, etc.), se si opera a tensione massima costante, e parametri di prova costanti, essa si può scrivere come $\Phi \approx \mathrm{N}_{\mathrm{i}} \mathrm{x} T$ facile anche da calcolare durante al fase di elaborazione 
delle acquisizioni. L'andamento della temperatura, evidenziato da Kaleta e Harig [5] e confermato da Curti et Al. [6,7], La Rosa e Risitano [8] e ultimamente adottato da M. Amiri e M.M. Khnsari [9], ha permesso di proporre metodi brevi per la determinazione del limite di fatica. L'osservazione sulla derivata nel tempo della temperatura già adottata da Curti et Al. [7] è stata ripresa ed adottata da M. Amiri e M.M. Khnsari in [9]. In tale lavoro e nel corrispondente brevetto, viene proposto anche un ulteriore metodo per la determinazione rapida del limite di fatica.

La costanza del parametro $\Phi=\int_{\mathrm{Ni}} T \operatorname{Tn}_{\mathrm{i}}$ è stata sperimentalmente sempre rilevata nella lunga attività di prove con acciai di diverso tipo e qualità da Risitano e dalla sua scuola [10]. Ciò conferma l'ipotesi di Feltner e Morrow sulla Energia limite a rottura come caratterizzante il materiale e di Taylor e Quinney [1] sulla costanza per gli acciai del fattore $\beta$.

Un'osservazione importante derivante dalla ormai vasta letteratura sull'argomento è che in prove di fatica monoassiali sia la pendenza della curva temperatura con numero di cicli che la temperatura di stabilizzazione sono funzione della sollecitazione applicata al provino (Fig. 3).
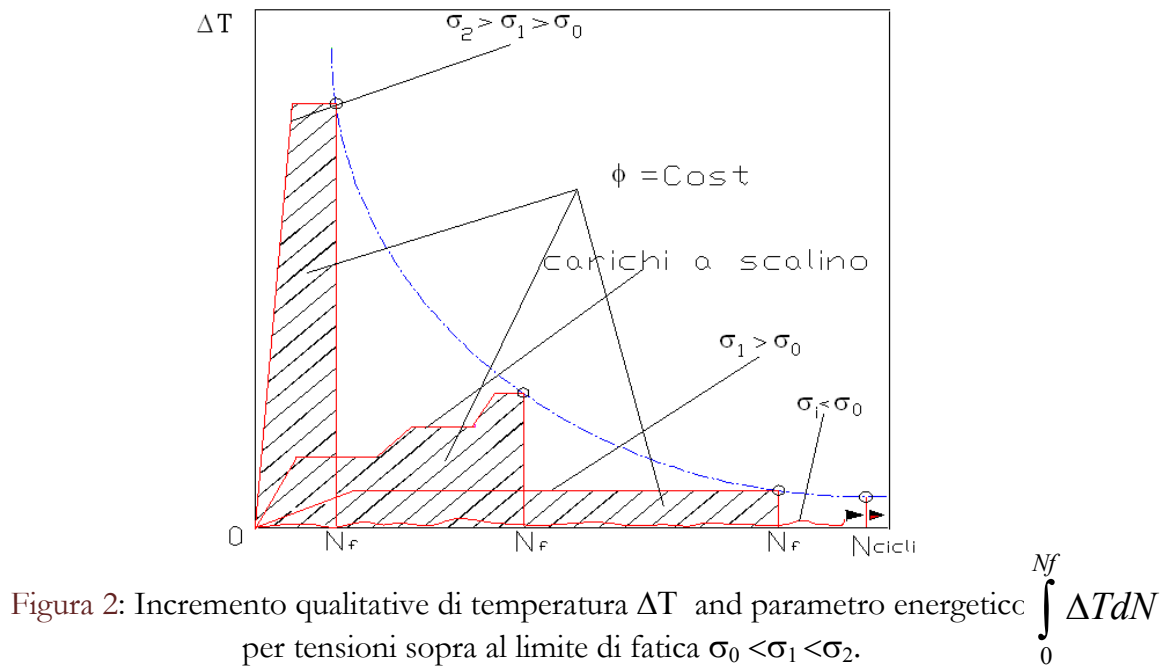

per tensioni sopra al limite di fatica $\sigma_{0}<\sigma_{1}<\sigma_{2}$

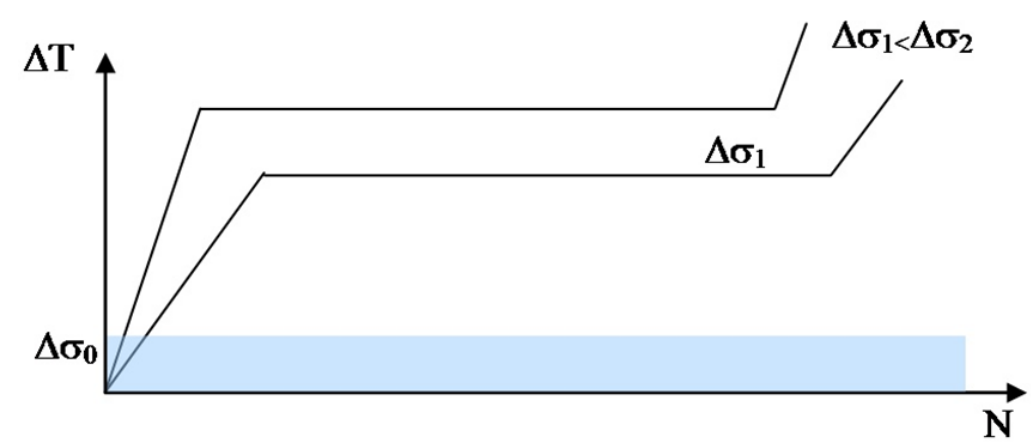

Figura 3: Incremento qualitativo delle temperature $\Delta$ T per tensioni sopra al limite di fatica $\Delta \sigma_{0}<\Delta \sigma_{1}<\Delta \sigma_{2}<\Delta \sigma_{3}$.

Come precedentemente detto, le diverse metodologie proposte per la ricerca del limite di fatica utilizzando il "terzo" parametro temperatura si basano sul fatto che l'incremento di temperatura è legato alla presenza di deformazioni plastiche permanenti che, con l'avanzare del numero di cicli, portano a rottura. Il limite di fatica per un materiale integro coincide con quel valore di sollecitazione limite che è capace di produrre anche fenomeni locali di plasticizzazione (Mason) per via dei quali, con il progredire del numero di cicli, si raggiungono i valori dell'Energia limite caratterizzante il provino. Se il materiale è danneggiato per una storia di carico precedente, il limite di fatica, inteso come quella tensione capace di produrre deformazioni plastiche locali che con il numero di cicli conducono alla rottura finale, risulta di valore più basso. In questo caso il fenomeno di deformazione plastica del provino è in parte iniziato e bastano valori di tensione minori per portare a rottura il provino, ovvero il limite di fatica risulta più basso. Dal punto di vista dell'energia in gioco e quindi della temperatura superficiale, essendo il materiale in una nuova zona di transizione (Mason), gli incrementi di temperatura superficiali sono limitati e difficili ancora da cogliere come incrementi significativi. Se però si applicano carichi elevati fuori dalla normale fascia di dispersione tipica delle prove di fatica, l'energia consumata per ciclo aumenta (modello di Feltener e Morrow, deformazioni unitarie plastiche maggiori) ed il valore di temperatura che si può leggere sul provino è superiore a 
quella che si avrebbe in un provino non danneggiato sollecitato alla stessa tensione. Quanto detto prima si può riscontrare sperimentalmente facendo riferimento alle esperienze dei ricercatori che hanno proposto le varie metodologie per la ricerca rapida del limite di fatica (Risitano, Luong, Curti, Blarasin, Amiri, etc.). Infatti, si vede che nelle curve di temperatura in funzione della tensione applicata a cui si rifanno i vari metodi, al diminuire del limite di fatica, corrispondono curve traslate verso l'asse delle ascisse con incrementi maggiori di temperatura a parità di sollecitazione applicata (vedi ad esempio Fig. 12 curva base e serie danneggiate).

Nel presente lavoro viene eseguita una verifica di quanto sopra detto e precisamente vengono rilevate le curve di temperatura per provini sollecitati a fatica con storie di carico diverse tali da produrre consumi di energia (deformazione plastica irreversibile) diversi. Per successive serie di carico affaticanti, viene determinato di volta in volta il nuovo limite di fatica e le relative curve a tempo.

\section{Procedura SPERIMENTALE E ANALISI DEI RISUltati}

$\mathrm{S}$ u provini in acciaio $\mathrm{C} 40$ sono state eseguite prove di fatica a frequenza di $10 \mathrm{~Hz}$ per rapporto di carico $\mathrm{R}=-1$. Per la caratterizzazione statica dell'acciaio in esame, sono state eseguite prove di trazione e i valori caratteristici mediati su tre provini sono riportati in Tab. 1. In Fig. 4 sono riportate la forma e le dimensioni dei provini utilizzati per le prove di fatica. Per tale acciaio si disponeva della curva di fatica definita in modo tradizionale da Berto e Lazzarin in [11].

\begin{tabular}{ccc}
\hline Ultimate strength & Yield strength & Fatigue limit \\
$\sigma_{\mathrm{R}}[\mathrm{MPa}]$ & $\sigma_{\mathrm{S}}[\mathrm{MPa}]$ & $\sigma_{0}(\mathrm{R}=-1)[\mathrm{MPa}]$ \\
660.00 & 510.00 & 251.8 by $[3]$ \\
\hline
\end{tabular}

Tabella 1: Comportamento meccanico dell'acciaio C40.

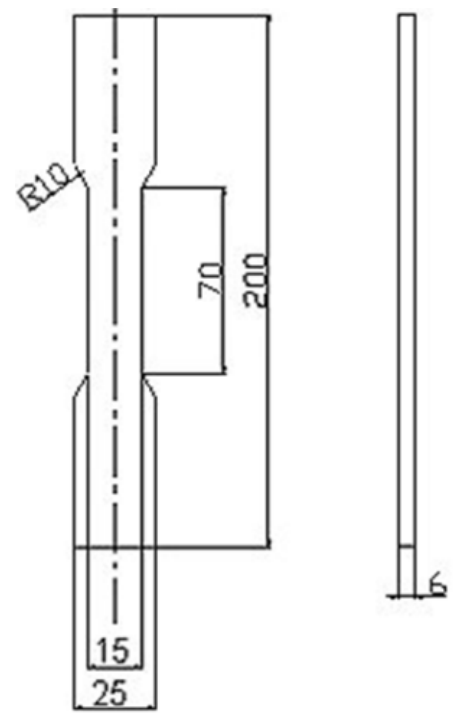

Figura 4: Dimensioni geometriche del provino ( $\mathrm{mm})$.

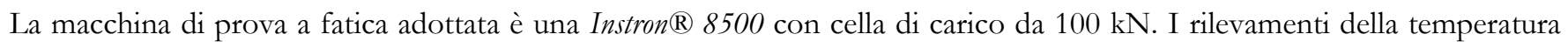
della superficie dei provini (opportunamente rivestiti di vernice nera con emissività $\varepsilon$ pari a 0.92), sono stati eseguiti mediante il termografo Agema Thermovision 900 con Thermovision $900 \mathrm{SW} / \mathrm{TE}$ scanner, con cui sono state rilevate e registrate le temperature superficiali di ciascun provino. Per ciascuna serie di prove, i provini usati erano in numero di tre. L'analisi della temperatura rilevata è stata eseguita alla fine di ciascuna prova ed i valori letti per ciascun provino sono stati fra di loro confrontati. Per la rappresentazione finale, dopo la valutazione della differenza che non superava mai il 3\% rispetto a quella media, per ragioni di chiarezza, si sono riportate solo le curve di temperatura a comportamento medio. La Tab. 2 riporta i valori dei carichi e delle sollecitazioni massime corrispondenti per ciascun step di carico di ciascuna serie (I, II, III) a cui sono stati sottoposti i provini.

Il programma di prove prevedeva l'esecuzione delle stesse in due distinte fasi. Nella Fase 1 con rapporto di carico $\mathrm{R}=-1 \mathrm{e}$ 
frequenza $10 \mathrm{~Hz}$, è stato caratterizzato (Termo-meccanica-catatterizzazione) il materiale con carichi a scalino (10.000 cicli per scalino) fino alla rottura del provino (Fig. 5) e rilevamento della temperatura superficiale per l'intero tempo di prova. L'analisi mediata dei dati (temperatura sul punto più caldo della superficie), su tre provini, permetteva la determinazione del limite di fatica e la valutazione del parametro energetico $\Phi=\int_{\mathrm{Nr}} \mathrm{TdN}$ attraverso cui, (metodo Risitano) veniva definita la curva di fatica del materiale integro.

\begin{tabular}{cc}
\hline$S[\mathrm{kN}]$ & $\sigma[\mathrm{MPa}]$ \\
14 & 155.5 \\
17 & 188.9 \\
20 & 222.2 \\
23 & 255.5 \\
26 & 288.9 \\
28 & 311.1 \\
\hline
\end{tabular}

Tabella 2: Valore dei carichi applicati.

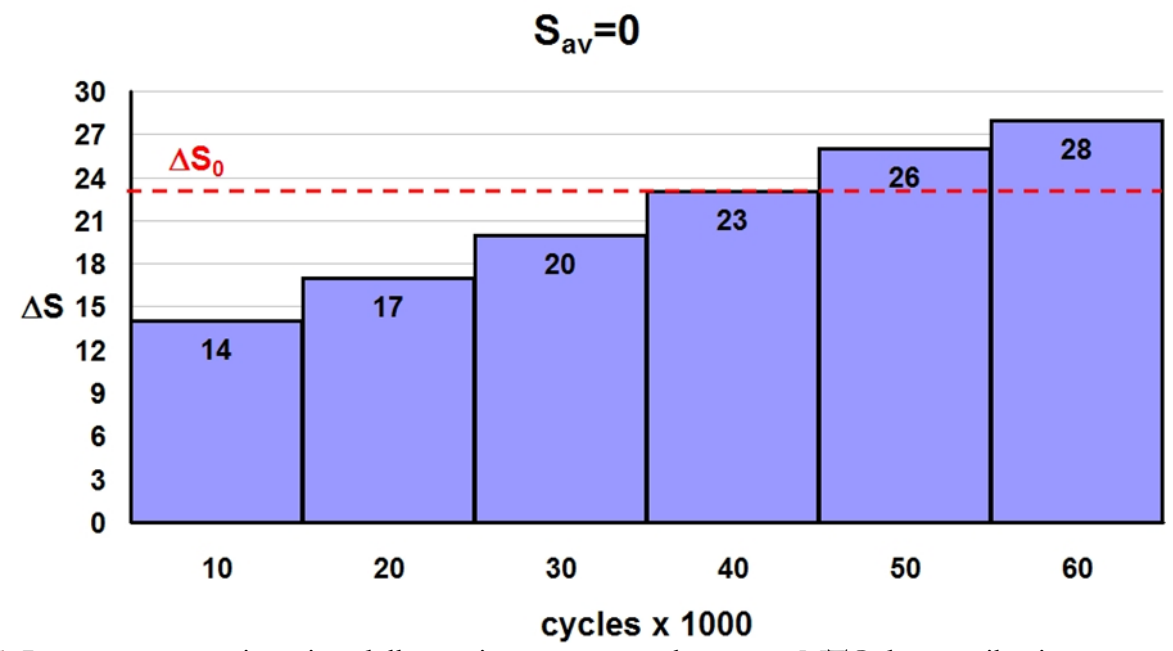

Figura 5: Incremento continuativo della tensione usato per la mappa MTC durante il primo stage di prove.

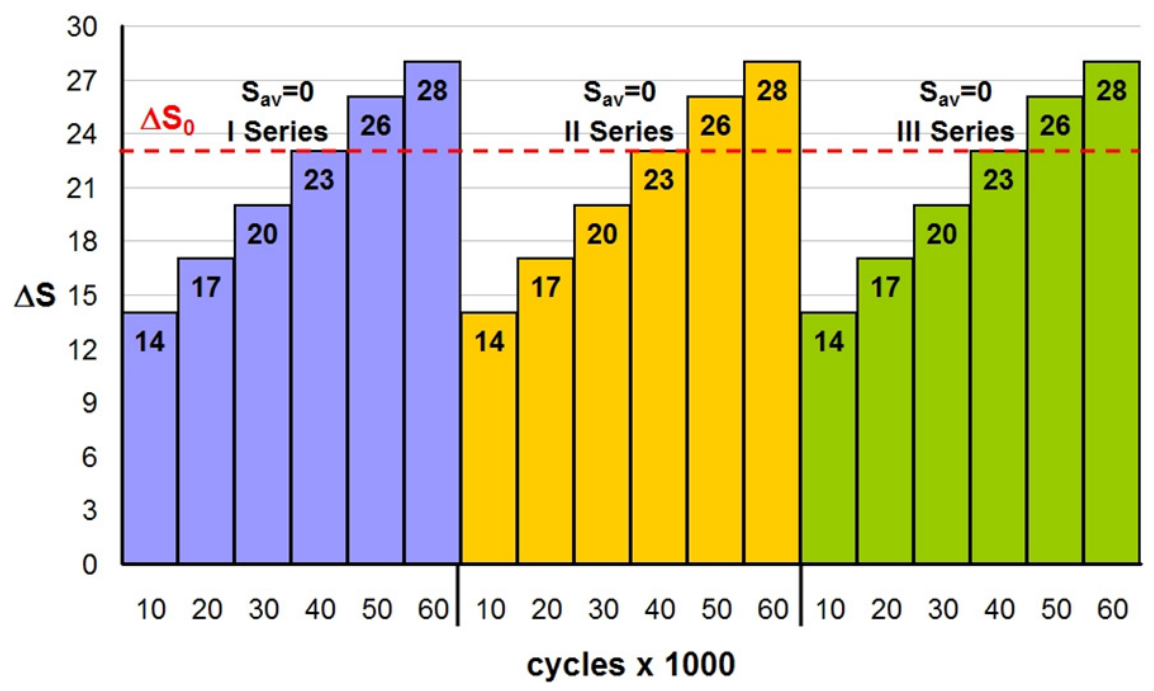

Figura 6: Successione degli step di carico usati durante il secondo stage di prove.

Nella Fase 2 (stesse condizioni di prova della Fase 1), si sono applicate tre successive serie di carico simili a quelle usate per la caratterizzazione del materiale (Fig. 6), senza arrivare a rottura, ma con carichi tali da produrre danno nel materiale. L'acquisizione della temperatura veniva eseguita per tutta la durata della prova. Essendo i carichi di valore superiore al 
limite di fatica del materiale, precedentemente determinato, essi erano idonei a generare microplasticizzazioni nel materiale con consumo di energia per deformazione plastica. Alla fine della Fase 2 si è stati in grado di avere l'andamento delle temperature per ciascun provino per le diverse serie di carico e, corrispondentemente, si è quantizzato il parametro energetico $\Phi$ parziale (alla fine di ciascuna serie applicata) e totale (a rottura). Con i dati acquisiti ed elaborati, alla fine di ciascuna serie, si è, quindi, determinato per ciascun provino il limite di fatica, la curva di Wöhler ed il parametro $\Phi=\int_{\mathrm{Ni}} T_{\mathrm{dn}}$, nonché il valore del parametro $\Phi\left(\Phi_{\mathrm{I}}, \Phi_{\mathrm{II}}, \Phi_{\mathrm{III}}\right)$. I dati riportati, così come le curve, si riferiscono, per ciascuna tipologia di prova, sempre ai valori di uno dei tre provini, in quanto i valori di temperatura rilevati erano fra loro molto vicini (le differenze di temperatura non superavano mai il 3\% ) e ai fini della elaborazione praticamente uguali.

\section{RISULTATI E COMMENTI}

$\mathrm{N}$ elle figure da 7 a 14, per la frequenza di prova $10 \mathrm{~Hz}$, sono riportati gli andamenti delle temperature mediate in funzione dei carichi applicati ai provini della fase 1 (provini lisci). Con i dati relativi alla Fig. 7 è stato determinato il limite di fatica (Fig. 8) che è risultato praticamente uguale a quello trovato da Berto e Lazzarin [11]. Nella Fig. 9, assieme alla curva di Wöhler tradizionale di [11], sono riportati i punti della curva di Wöhler definiti per il valore del parametro $\Phi=\mathrm{N}_{\mathrm{ixT}}$ (circa pari all'integrale dell'area sottesa alla curva delle temperature) che è risultati pari a 110x104 Cicli $\mathrm{x}{ }^{\circ} \mathrm{C}$.

Le figure da 8 a 14 si riferiscono ai risultati della fase 2 di prova. Il post processing dei dati acquisiti ha evidenziato che il punto più caldo della superficie del provino non cambiava dopo l'applicazione delle successive serie di carico e fino alla rottura del provino. In Fig. 10 è riportato l'andamento delle temperature per tutte le serie di carichi applicati ai provini. Nella Fig. 11, per meglio evidenziare il fenomeno, è riportato l'andamento delle temperature, a parità di carico applicato al provino, ma appartenente a serie di carico successive (I, II, III). L'esame delle figure evidenzia:

1) l'andamento della temperatura relativa alla prima serie di carichi, coincide nei limiti della normale dispersione, con quello di Fig. 7 relativo ai provini integri, che, tuttavia, sono comunque diversi;

2) i valori di temperatura che si rilevano per sollecitazioni uguali, ma di serie successive diverse, sono sempre più elevati man mano che si passa dalla serie (I) alla seconda (II) e alla terza (III);

3) la differenza di temperatura, a parità di carico, per serie diverse è tanto più alto quanto più elevato è il valore del carico applicato.

In Fig. 12 sono riportate le temperature di stabilizzazione per ciascun valore di tensione applicata da cui secondo Curti e Risitano [7] si giunge alla determinazione del limite di fatica.

Nella Fig. 13 sono riportate la curve di Wöhler definite attraverso il parametro $\Phi$, calcolato per tutta la prova, e dei parametri $\Phi_{i}$ parziale per ciascuna serie. In particolare le curve di Wöhler successive (spostate in basso nella figura) sono state costruite facendo, di volta in volta, riferimento alla differenza fra il parametro $\Phi$ totale e il parametro $\Phi_{i}$ parziale precedente (energia "consumata"). In Tab. 4 sono riportati i valori dei parametri $\Phi$ parziali e totali. Nella stessa Fig. 13 è riportata anche la curva di Wöhler definita da Berto \& Lazzarin in [11]. I risultati, sintetizzati nelle figure sopra descritte, evidenziano ancora che il limite di fatica cambia man mano che al provino vengono applicate le serie successive di carichi. Nella Fig. 14, seguendo in parte quanto indicato da Miner-Manson, sono riportate le rette che uniscono i punti relativi ai limiti di fatica (ascisse $\mathrm{N}=2 \times 10^{6}$ ) dopo l'applicazione della I serie di carichi e della I e II serie di carichi, con la tensione di snervamento del materiale. Si può osservare come i punti determinati attraverso il parametro energetico $\Phi$ siano allineati e posizionati su tali rette.

\begin{tabular}{ccc}
\hline$f[\mathrm{~Hz}]$ & $S_{0}[\mathrm{KN}]$ & $\sigma_{0}[\mathrm{MPa}]$ \\
10 & 24.2 & 258.60 \\
\hline
\end{tabular}

Tabella 3: Carichi corrispondenti al limite di fatica per l'acciaio C40 tramite TMC.

\begin{tabular}{cc}
\hline$\left[\right.$ Ciclix $\left.^{\circ} \mathrm{C}\right]$ & $\mathrm{R}=-1 f=10 \mathrm{~Hz}$ \\
$\Phi_{I}$ & $12.2^{*} 10^{4}$ \\
$\Phi_{I I}$ & $49.6 * 10^{4}$ \\
$\Phi_{I I I}$ & $52.7 * 10^{4}$ \\
$\Phi$ & $114.5^{*} 10^{4}$ \\
\hline
\end{tabular}

Tabella 4: Parametro energetico $\Phi$. 


\section{INDICAZIONI DERIVANTI DALL'ANALISI TERMICA}

ome è noto, seguendo la regola del Miner, il danno cumulativo è definito da $\mathrm{D}^{\mathrm{M}}=\Sigma_{\mathrm{i}} \mathrm{n}_{\mathrm{i}} / \mathrm{N}_{\mathrm{i}}$, in cui, $\mathrm{n}_{\mathrm{i}}$ è il numero di cicli alla tensione $\sigma_{\mathrm{i}}, \mathrm{e} \mathrm{N}_{\mathrm{i}}$ è il numero di cicli a rottura per quella tensione. Pertanto, nel nostro caso, dopo la prima serie di carichi risulta:

$\mathrm{D}^{\mathrm{M}} \cong \mathrm{n}_{1} / \mathrm{N}_{1}+\mathrm{n}_{2} / \mathrm{N}_{2}+\mathrm{n}_{3} / \mathrm{N}_{3}+\mathrm{n}_{4} / \mathrm{N}_{4}+\mathrm{n}_{5} / \mathrm{N}_{5}$

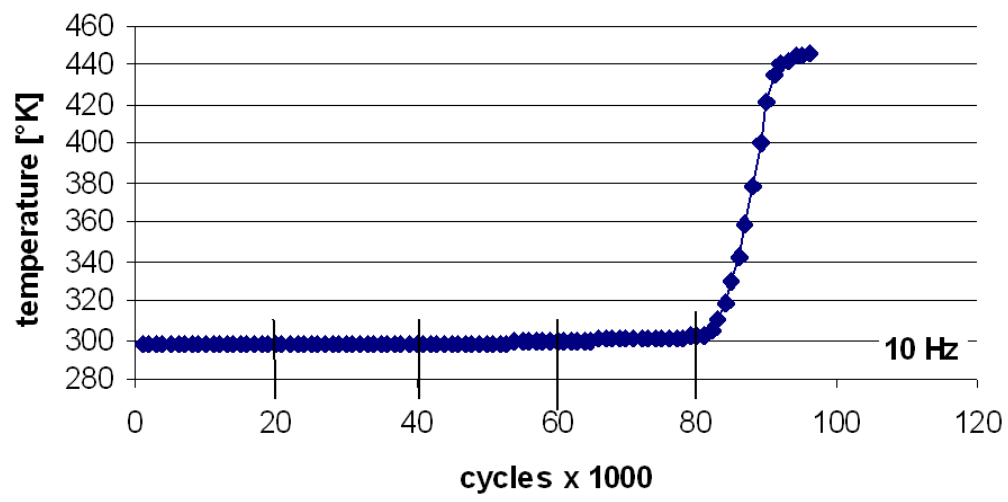

Figura 7: Incrementi di temperature $\mathrm{T}$ in funzione dei numeri di cicli per l'acciaio C40 con frequenze di prova di $10 \mathrm{~Hz}$.

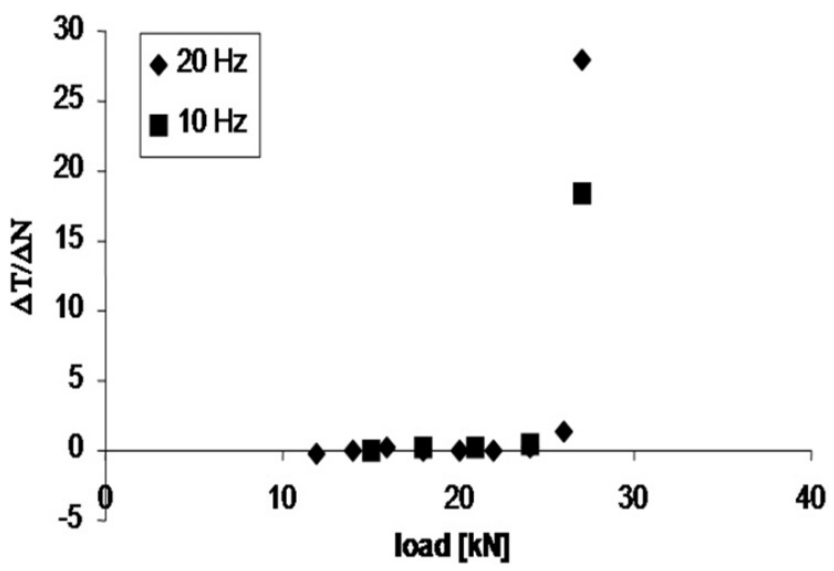

Figura 8: Determinazione del limite di fatica $\Delta \sigma_{0}=\mathrm{S}_{0} / \mathrm{A}$ con il metodo Risitano.

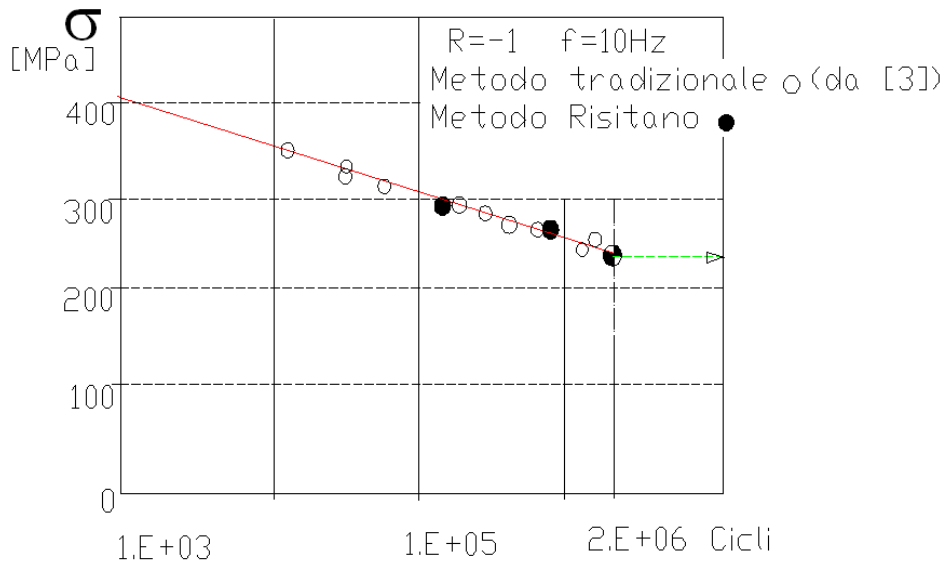

Figura 9: Curva di Wöhler per provini lisci ricavata con metodo tradizionale e con quello termografico per frequenza di prova di $10 \mathrm{~Hz}$ e rapporto di carico $\mathrm{R}=-1$. 


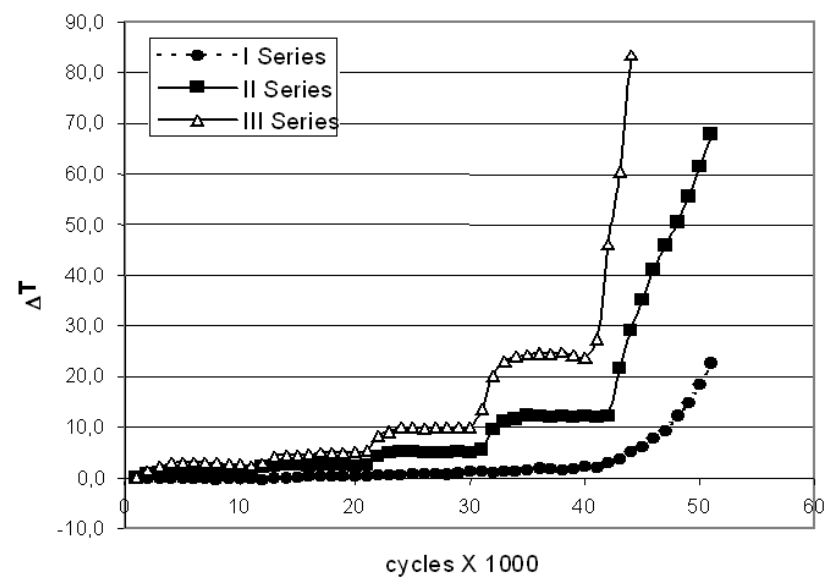

Figura 10: Incremento di temperatura $\mathrm{T}$ in funzione del numero di cicli per acciaio C40 (f=10 Hz, R=-1) per differenti serie (I, II, III).
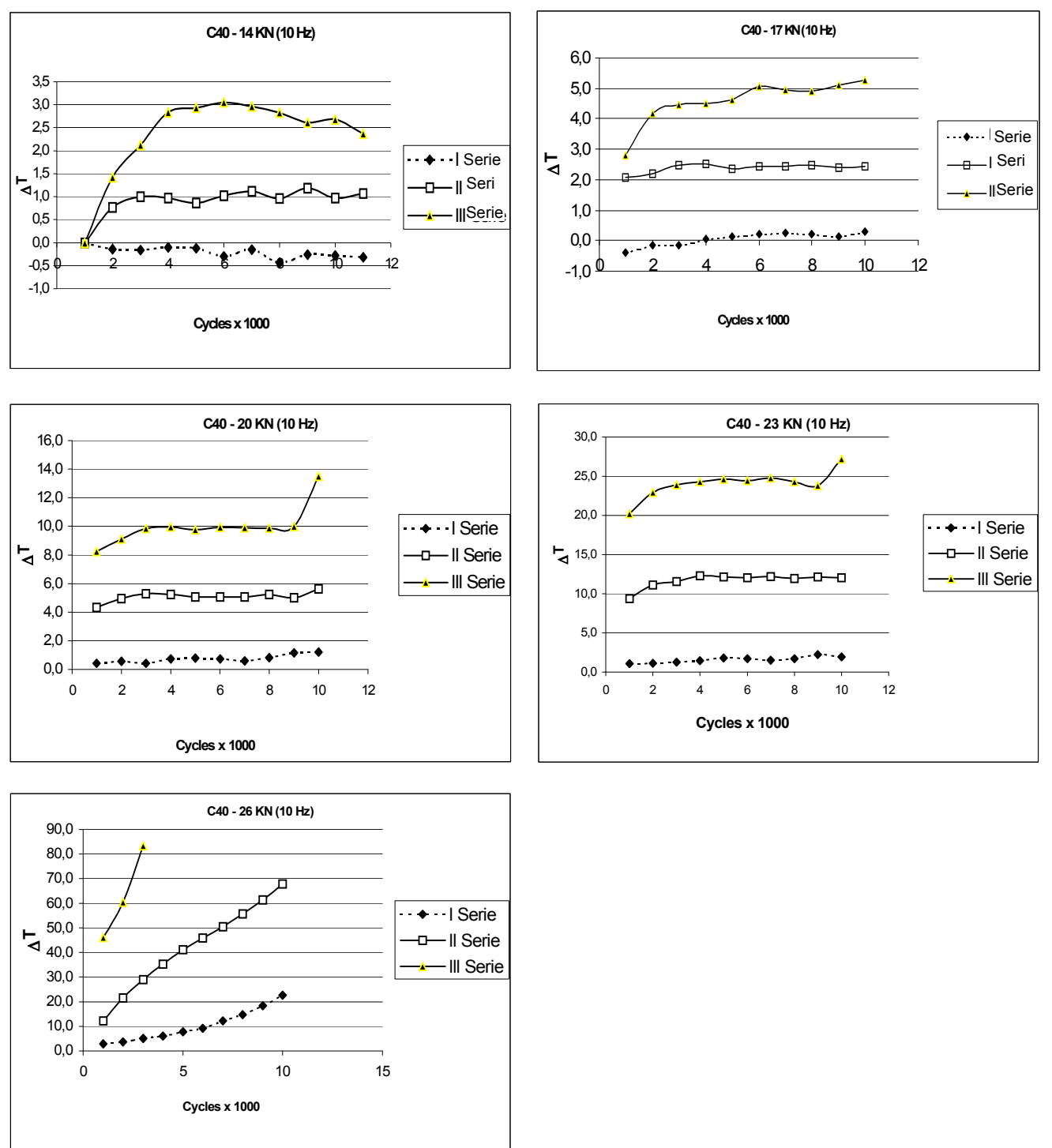

Figura 11: Andamenti del $\Delta \mathrm{T}$ di temperature in funzione del numero di cicli ( $\mathrm{f}=10 \mathrm{~Hz}, \mathrm{R}=-1$ ) per differenti serie (I, II, III). 


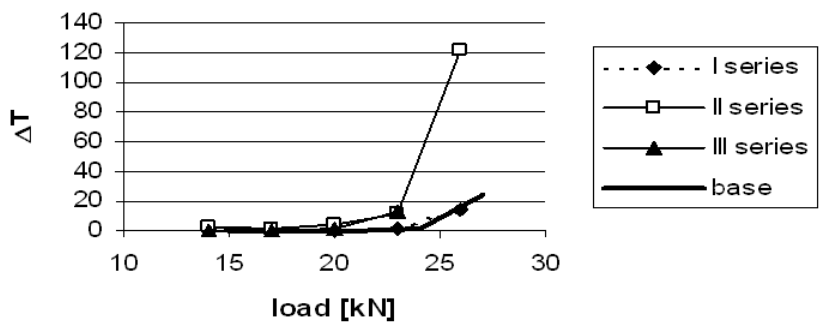

Figura 12: Determinazione del limite di fatica $\Delta \sigma_{0}=\mathrm{S}_{0} / \mathrm{A}$ con il metodo Risitano.

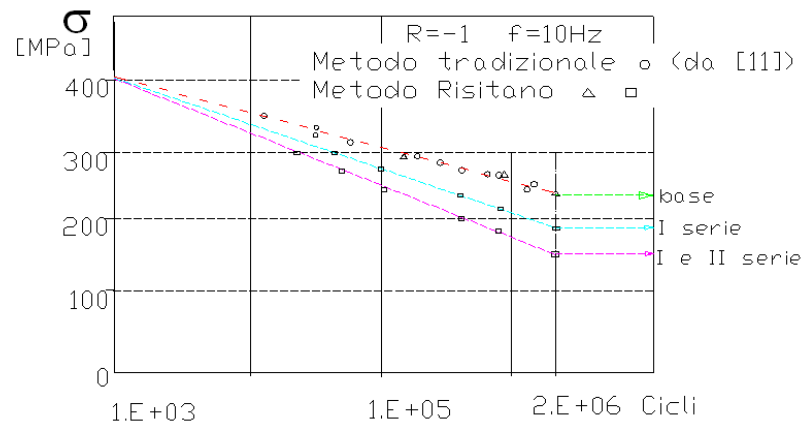

Figura 13: Curve di Wöhler di provini lisci (base) e di provini sottoposti alla prime I e alla I e II serie di carichi definite secondo il metodo Risitano

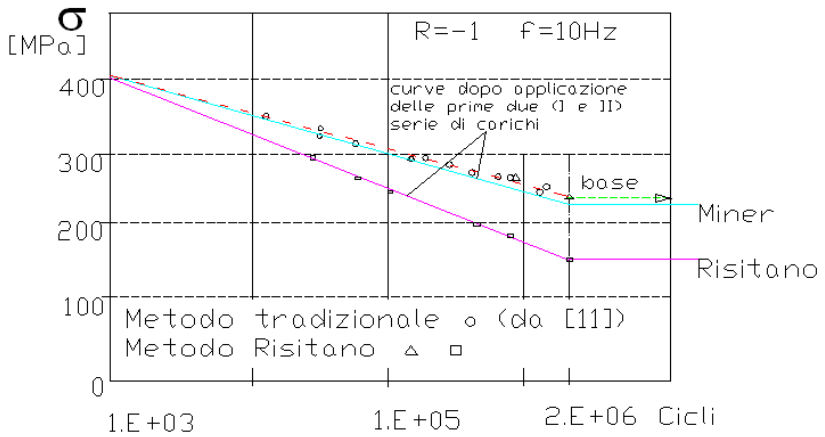

Figura 14: Curve di Wöhler di provini lisci (base) e di provini sottoposti alle prime due serie di carichi secondo Miner e Risitano.

Facendo riferimento al metodo Risitano e quindi al parametro energetico $\Phi$, il danno risulta:

$$
\mathrm{D}^{\mathrm{I}}=\Phi^{\mathrm{I}} / \Phi \cong \sum_{\mathrm{i}}\left(\mathrm{n}_{\mathrm{i}} \Delta \mathrm{T}_{\mathrm{i}} / \mathrm{N}_{\mathrm{i}} \Delta \mathrm{T}_{\mathrm{i}}\right)
$$

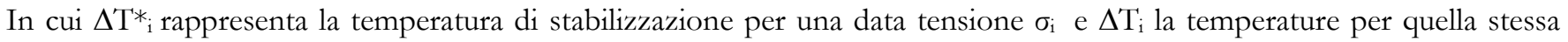
tensione con cui il provino non danneggiato arriverebbe a rottura $\left(\Delta \mathrm{T}_{\mathrm{i}} \mathrm{NN}_{\mathrm{i}}=\right.$ Cost $)$.

Esplicitando, nel nostro caso si ha:

$$
\mathrm{D}^{\mathrm{I}}=\Phi^{\mathrm{I}} / \Phi \cong\left(\mathrm{n}_{1} \Delta \mathrm{T}^{*} / \mathrm{N}_{1} \Delta \mathrm{T}_{1}+\mathrm{n}_{2} \Delta \mathrm{T}^{*} / \mathrm{N}_{2} \Delta \mathrm{T}_{2}+\mathrm{n}_{3} \Delta \mathrm{T}^{*} / \mathrm{N}_{3} \Delta \mathrm{T}_{3}+\mathrm{n}_{4} \Delta \mathrm{T}^{*} / \mathrm{N}_{4} \Delta \mathrm{T}_{4}+\mathrm{n}_{5} \Delta \mathrm{T}^{*} / \mathrm{N}_{5} \Delta \mathrm{T}_{5}\right)
$$

essendo, $\Phi=$ costante e pari a $\mathrm{N}_{1} \Delta \mathrm{T}_{1}=\mathrm{N}_{2} \Delta \mathrm{T}_{2}=\mathrm{N}_{3} \Delta \mathrm{T}_{3} \ldots$ (energia limite di deformazione plastica costante del materiale)

Essendo, come verificato sperimentalmente, i rapporti di temperatura, a parità di carico, per provini danneggiati e non, maggiori di 1, risulta che il danno valutato secondo la ipotesi del Miner è inferiore a quello valutato sulla base dell'energia spesa per ciclo. Tuttavia, nel caso in cui il provino già danneggiato, venisse portato a rottura con carichi di poco superiori al limite di fatica, i rapporti di temperatura sarebbero prossimi all'unità e l'ipotesi del Miner coinciderebbe con quella derivante dal tener conto dell'effetto energetico ( $\left.\mathrm{D}^{\mathrm{M}} \cong \mathrm{D}^{\mathrm{I}}\right)$. In casi diversi, ovvero, quando venissero applicati carichi elevati per arrivare a rottura, applicando l'ipotesi del Miner, si avrebbero, sottostime del danno con errori anche 
significativi. Tale osservazione conferma quanto riportato da Iqban Rasool Memon ed Al. [12], per cui, se si inverte l'ampiezza di carichi affaticanti (superiori al limite di fatica) la regola del Miner per la valutazione del danno non può essere usata. L'applicazione di quanto detto alla tipologia di carichi adottati nel lavoro di Iqban Rasool Memon, porterebbe in un senso (carichi crescenti) ad un valore di danno pari a:

$$
\mathrm{D}^{\mathrm{M}}=\mathrm{n}_{1} / \mathrm{N}_{1}+\mathrm{n}_{2} / \mathrm{N}_{2}
$$

ed ad un valore, in termini energetici, pari a:

$$
\mathrm{D} \cong \Phi \mathrm{I} / \Phi \cong \mathrm{n}_{1} \Delta \mathrm{T} *_{1} / \mathrm{N}_{1} \Delta \mathrm{T}_{1}+\mathrm{n}_{2} \Delta \mathrm{T} *_{2} / \mathrm{N}_{2} \Delta \mathrm{T}_{2}
$$

Se l'ampiezza del primo carico fosse di poco superiore al limite di fatica ed il secondo fosse di parecchio più elevato, si avrebbe $\Delta \mathrm{T}^{*}{ }_{1}=\Delta \mathrm{T}_{1}$ ed, essendo stato il materiale poco danneggiato, $\Delta \mathrm{T}^{*} \cong \Delta \mathrm{T}_{2}$ e quindi $\mathrm{D}^{\mathrm{M}} \cong \mathrm{D}^{\mathrm{I}}$. Applicando i carichi in senso opposto (carichi decrescenti) si avrebbe $\Delta \mathrm{T}_{2}{ }_{2}=\Delta \mathrm{T}_{2}$ ma una $\Delta \mathrm{T}^{*}{ }_{1}$ completamente diversa e maggiore da $\Delta \mathrm{T}_{1}$ (il materiale è stato danneggiato). Di conseguenza sarebbe $\mathrm{D}^{\mathrm{M}} \cong \mathrm{n}_{2} / \mathrm{N}_{2}+\mathrm{n}_{1} / \mathrm{N}_{1}$ pari a quello di prima (carichi crescenti) ma $\mathrm{D}^{\mathrm{I}} \cong \Phi^{\mathrm{I}} / \Phi \cong \mathrm{n}_{2} \Delta \mathrm{T}^{*} / \mathrm{N}_{2} \Delta \mathrm{T}_{2} \mathrm{n}_{1}+\Delta \mathrm{T}^{*}{ }_{1} / \mathrm{N}_{1} \Delta \mathrm{T}_{1}$ decisamente più alto.

Come già riferito in [49] vale la linearità del danno e la curva a tempo si può ottenere, una volta determinato il limite di fatica, tracciando nel diagramma semilogartmico $(\sigma \log \mathrm{N})$ la retta che congiunge il punto di coordinate $\sigma_{0}$ e $2 \times 10^{6} \mathrm{Cicli}$, con il punto di ascisse 0 e ordinata $\sigma_{\mathrm{s}}$ (tensione di snervamento). Tale curva, tuttavia, non sempre coincide con quella tracciata secondo le indicazioni del Miner, specialmente quando la storia di carico è tale che tensioni, apparentemente sotto il limite di fatica del materiale, diventano affaticanti per il danno prodotto da uno di essi (magari ad ampiezza elevata) applicato in precedenza. Il diagramma di Fig. 11, in cui sono stati riportati gli andamenti delle temperature a parità di tensione applicata nelle successive serie di carico, mette in evidenza questo aspetto. Se si facesse riferimento al limite di fatica del materiale non danneggiato $\sigma_{0}=251,8 \mathrm{~N} / \mathrm{mm}^{2}$, in tutta la storia di carichi applicati solo con i carichi 23 e $26 \mathrm{kN}$ si raggiungerebbero tensioni affaticanti (carico/area sezione provino) pari a 255,6 e 288,9 N/PPDIn realtà, dopo l'applicazione della prima serie, che riporta un incremento di temperatura già per $17 \mathrm{kN}$ e, di conseguenza, ad un limite di fatica differente (in Fig. 12, la curva "base" è riferita alla curva relativa ai provini della Fase 1), si avrebbero, per i carichi successivi (della stessa serie), gradi di danneggiamento sempre maggiori. Questo è ancora più accentuato dai salti di temperatura, sempre più ampi a parità di tensione applicata per serie successive (Fig. 11). In altre parole, con l'applicazione della successiva serie (II serie di carichi), la riduzione del limite di fatica, anche per l'effetto della prima serie, amplifica il fenomeno termico. L'esame della figura evidenzia ancora come, all'applicazione della III serie di carichi anche quei carichi, che in partenza erano inferiori al limite di fatica del materiale diventino affaticanti ed in grado di contribuire al consumo di energia plastica. I rapporti $\Delta \mathrm{T}_{\mathrm{i}} / \Delta \mathrm{Ti}$, derivanti dall'avere messo in gioco parametri energetici, permette di evidenziare e definire il danno cumulativo come rapporto della perdita di energia per ogni ciclo rispetto all'energia limite $\mathrm{E}_{\mathrm{c}}$.

Quanto precedentemente detto è sintetizzato in Fig. 14 in cui, assieme alla curva di Wöhler tradizionale (per provini lisci), è stata riportata la retta (Miner in figura) che si ottiene attraverso la regola del Miner-Manson dopo l'applicazione delle prime due serie di carichi. Essa, per il fatto che praticamente solo due dei carichi applicati superano il limite di fatica del materiale, si dispone molto vicina alla curva base. Nella stessa figura è riportata la curva di Wöhler (Risitano in figura) definita con il metodo Risitano, ovvero, facendo riferimento al parametro energetico e quindi alla energia persa per ciclo per effetto di ciascuno dei carichi applicati (quelli della seconda serie a partire dal valore di $17 \mathrm{kN}$ provocano danno ed il rapporto del $\mathrm{n}_{\mathrm{i}} / \mathrm{N}_{\mathrm{i}}$ va corretto con i rapporti di temperatura $\left.\Delta \mathrm{T}_{\mathrm{i}} / \Delta \mathrm{Ti}\right)$.

\section{CONCLUSIONI}

$\mathrm{V}$

engono messe in evidenza alcuni aspetti che hanno portato al lavoro degli autori [13] sulla valutazione del danno in materiali o componenti meccanici. Utilizzando gli stessi dati sperimentali di [13] sono state fatte alcune considerazioni sulla possibilità della valutazione del danno sulla base di parametri che tengano conto della energia persa per ciclo.

I risultati delle prove di fatica, effettuate su provini di acciaio C40 danneggiati con applicazioni di serie di carichi successive e per cui è stata costantemente rilevata la temperatura durante tutta la prova, hanno messo in evidenza quanto segue:

1) Il limite di fatica del materiale, come è ovvio, varia con il grado del danno (energia spesa/energia limite) ed è facilmente rilevabile mediante l'analisi termica sotto carico;

2) la temperatura del punto più caldo della superficie del provino sollecitato a fatica varia per valori di carico uguali 
appartenenti a serie di carico successive;

3) durante l'applicazione delle successive serie di carico, il punto, in cui si rileva la massima temperatura, rimane lo stesso fino alla completa rottura del provino;

4) la legge lineare del danno, per la ricerca delle curve a tempo, può essere sempre applicata se si parte dalla valutazione esatta del nuovo limite di fatica;

5) viene confermata la legge lineare cumulativa del danno, ma la stessa legge scritta in termini di energia "consumata" per ciclo, evidenzia che la legge del Miner in accordo con quanto segnalato da Iqban Rasool Memon et Al. [12] non sempre può essere applicata;

6) le variazioni di temperatura rilevate per tensioni di uguale entità, ma applicate dopo che il materiale è stato danneggiato "diversamente", sono tanto più elevate quanto più è grande il grado di danno.

Le osservazioni sopra riportate, basate di fatto su considerazioni energetiche, insieme a quanto già riferito dagli autori in [13] e [14], permettono di eseguire in modo semplice valutazioni del danno nei materiali ed in componenti meccanici. Gli autori considerano osservazioni preliminari quelle sui limiti di validità della legge del Miner segnalati anche in [12] e si propongono di approfondirle con adeguate indagini che possano mettere in conto l'energia rilasciata per effetto del "danno".

\section{BIBLIOGRAFIA}

[1] G. I. Taylor, H. Quinney, In: Proc. R. Soc., A 134 (1934) 307.

[2] J. J. Mason, A. J. Rosakis, G. Ravichandra, Mech. Mat., 17 (1994) 135.

[3] C.E. Feltner, J.D. Morrow, Trans. ASME, Ser. D: J Basic Eng, 83 (1961) 15.

[4] D. Dengel, H. Harig, Fatigue Fract Eng Mater Struct, 3 (1980) 113.

[5] J. Kaleta, R. Blotny, H. Harig, J Test Eval, 19 (1990) 326.

[6] G. Curti, G. La Rosa, M. Orlando, A. Risitano, In: 14 ${ }^{\text {th }}$ AIAS Italian National Conference, Catania, Italy, (1986) 211.

[7] G. Curti, A. Geraci, A. Risitano, ATA Ingegneria Automotoristica, 10 (1989) 634.

[8] G. La Rosa, A. Risitano, International Journal of Fatigue, 22 (2000) 65.

[9] M. Amiri, M.M. Khonsari, International Journal of Fatigue, 32 (2010) 382.

[10] G. Fargione, A. Geraci, G. La Rosa, A. Risitano, International Journal of Fatigue, 24 (2002) 11.

[11] F. Berto, P. Lazzarin ,La Metallurgia Italiana, 3 (2005) 23.

[12] Iqbal Rasool Memon, Xing Zhang, Deyu Cui, International Journal of Fatigue, 24 (2002) 29.

[13] A. Risitano, G. Risitano, In: Workshop IGF, Forni di Sopra (2010).

[14] A. Risitano, G. Risitano; In: Workshop IGF, Forni di Sopra (2009) e pubblicato su "Frattura ed Integrità Strutturale", 9 (2009) 123.

[15] G. C. Sih, Multiscale fatigue crack initiation and propagation of engineering materials, structural integrity and microstructural worthiness: solid mathematics and its applications, Springer, 152 (2008).

[16] A. Palmgren, Die Lebensdauer von Kugellagern, Verfahrenstechinik, Berlin, 68 (1924) 339.

[17] M. A. Miner, Journal of Applied Mechanics, 67 (1945) A159.

[17] L. Locati, Metall. It. Al., 27 (1935) 188.

[18] M. Prot, Misure et control, 13 (1948) 301.

[19] M. Prot, WADCTR (1952) 53.

[20] O. Föppl, V.D.I.Z., 70(39) (1926) 1291.

[21] O. Föppl, P. Ludwik, V.D.I.Z., 76(14) (1932) 683.

[22] J F Delorme, G Sinicki, P. Gobin, J. Phys. D: Appl. Phys., 1 (1968) 1737.

[23] T. Catalbiano, A. Geraci, M. Orlando, Il Progettista Industriale, (1984) 2.

[24] A. Geraci, G. La Rosa, A. Risitano, In: CRES Symposium, Catania Italy, 1984, pubblicato in ATA Ingegneria Automotoristica 38 (1985) 8.

[25] M.P. Luong, Mech. Mater., 28 (1988) 155.

[26] M.P. Luong, SPIE, 1682 (1992) 222.

[27] M.P. Luong, K Dang-Van, In: Atti della fondazione G. Ronchi, IL (1993) .

[28] M.P. Luong, Nucl. Eng. Des., 158 (1995) 363.

[29] A. Blarasin, R. Fracchia, M. Pozzati, ATA - Ingegneria Automotoristica, 51 (5) (1988) 255.

[30] F. Curà, G. Curti, R. Sesana, Int. J. Fatigue 27 (2005) 453.

[31] F. Walter, D.Eifler, Journal of Solid Mechanics and Materials Engineering, 2 (4), 2008. 
[32] A. Geraci, G. La Rosa, A. Risitano, In: $7^{\text {th }}$ International conference on mechanical behaviour of material, The Hague (1995).

[33] R. Blonty, Kaleta, Analysis of Mathematical model of the Fatigue Energy Hypothesis as seen by identification theory, $\mathrm{PhD}$ Thesis, Inst. of material Science and Appl. Mechanics, TU Wroclaw, 199 (1978).

[34] R. Blonty, Kaleta, International Journal of Fatigue, 1 (1986) 35.

[35] R. Blonty, Kaleta, In: 7th International Conference on fracture (ICF-7), Houston Tex., Pergamon Press, Oxford, (1989) 1195.

[36] B. Atzori, E. Gasparini, G. Meneghetti, In: Proceedings of 30th AIAS national conference, (2001) 367.

[37] B. Atzori, G. Meneghetti, LCF 5. In: Proceedings of the $5^{\text {th }}$ international conference on low cycle fatigue, P.D. Portella, H. Sehitoglu and K. Hatanaka, Editors, DVM, Berlin (2003) 147.

[38] G. Meneghetti, International Journal of Fatigue, 29(1) (2007) 81.

[39] O.A. Plenkev, N. Saintier, T. Palinluc, S.V. Uranov, Naimark, Material Science and Engineering, 462(1-2) (2007) 367.

[40] N.W. Klingbeil, Int. J. Fatigue, 25 (2003) 117.

[41] T. Boulanger, A. Chrysochoos, C. Mabru, A. Galtier, Int. J. Fatigue, 26 (2004) 221.

[42] N. Ranc D. Wagner, P.C. Paris, Study of thermal effects associated with crack propagation during very high cycle fatigue tests, Acta Materialia, 56(15) (2008) 4012.

[43] M. Selek, Ö. S. Şahin, Ş. Kahramanl, In: EUROCON 2007 The International Conference on "Computer as a Tool”" Warsaw (2007).

[44] P. Lazzarin, P. Livieri, F. Berto, et alii, Engineering fracture mechanics, 75(7) (2008) 1875.

[45] A. Chrysochoos, H. Louche, International Journal of engineering science, 38 (2000) 1759.

[46] ASTM E 466-72 Standard practice for conducting constant amplitude axial test of metallic materials.

[47] J. Hoodwany, G. Ravichandran, A. J. Rosakis, P. Rosakis, Experimental Mechanics, 40(2) (2000).

[48] A. Chrysochoos, B.Berthel, F. Latourte, S. Pagano, B Wattrisse, B. Weber, Strain, 44 (2008) 327.

[49] D. Macduogall, Experimental Mechanics, 40 (3) (2000).

[50] F. Walter, D. Eifler, Journal of Solid Mechanics and Material Engineering, 2(4) (2008). 